\title{
Tell Me What You Want, What You Really, Really Want: Understanding User Perspectives with Comparative Analysis
}

\author{
Zoe Chao \\ Penn State University, USA \\ Introduction \\ Comparative Analysis
}

Comparative analysis, or, as the industry calls it, "competitive analysis," has always been a common practice in evaluating usability. By comparing features with competitors, we can determine the product's strengths and weaknesses. In his usability engineering model, Nielsen stated that existing products are often the best prototypes at the predesign stage because they are already fully developed. ${ }^{1}$ So even before building the product, valuable information about what features support user needs and meet user expectations can be collected. ${ }^{2}$ Useful features can be incorporated into the new product and potential usability problems can be avoided. ${ }^{3}$

Libraries have done comparative analyses with products and systems, particularly on online catalogues and discovery tools. Asher, Duke, and Wilson compared the search results of EBSCO Discovery Services (EDS), Summon by Serial Solutions, Google Scholar, and conventional library resources. ${ }^{4}$ In the study, participants were asked to select resources needed for fictitious assignments using two of these platforms. Their selections were then scored by librarians in terms of scope, relevancy, reliability, etc. Majors also conducted comparative usability testing of vendor-provided discovery interfaces (Encore Synergy, EDS, Primo Central, Summon, and WorldCat Local). ${ }^{5}$ Similarly, he used task-based testing to assess user experience. However, each participant tested only one interface to prevent "learning" from previous experience. The systems were compared regarding the issues encountered during the tests. Both studies focused on comparing products' usefulness and usability.

\section{Usability and User Experience}

Usability of a product has been the focus in UX studies for more than three decades. ${ }^{6}$ The usability testing method that has participants think aloud as they complete tasks has been widely adopted since 1990 s. $^{7}$ The objective measurements of task success and task completion time from usability testing are often the metrics used in comparative analysis. ${ }^{8}$

In the 2000s, the new term "user experience" (UX) began to appear in the research and became an encompassing phrase for all experiences users have when interacting with an interactive product. ${ }^{9}$ Researchers came to realize that solely focusing on usability has its limitations. Hassenzahl and Tractinsky in User experience-a research agenda point out that goal-oriented usability testing evaluates only the product's pragmatic, instrumental value. The hedonic, affective value of UX is equally essential for a product. ${ }^{10}$ The ever-evolving technology and competitive digital market have proved that, more than usefulness and usability, desirability is what makes a product, well, desirable. Users' cognition matters, as does a user's affect and sensation. ${ }^{11}$

As user experience (UX) gains traction in libraries, the focus of our research has shifted from simple usability to a broader understanding of user perspectives. A library's online presence is no longer only about usability and findability, but about connecting to users and understanding their needs. Contrasted with the straightforward approach of measuring task performance in usability testing, how do we evaluate other dimensions of user experience of a system? In this paper, I will discuss how to use comparative analysis to understand the user's context and preferences for the library's online environment. 


\section{Study Overview}

From fall 2016 to spring 2018, 186 small-scale UX tests were conducted at the Penn State University's main library entrance. Participants spent five to ten minutes completing one or two tasks in exchange for a cup of coffee and a snack. In this setting, called the UX Café, different UX methods were used in addition to usability testing. The pre-fab, low-cost format of the UX Café allows the libraries to conduct lightweight and focused UX studies regularly without the overhead of recruitment and scheduling in the traditional usability studies process. All the comparative analysis testing was conducted at the UX Café.

Before diving into which "competitor products" to measure against, a scope of the study was first determined and one usability task was designed based on the defined scope. The point of one single definite task was to make sure that participants were not overwhelmed by testing with multiple websites during the short timeframe of the UX Café. The tasks were user-centered and scenario-based: for example, "Imagine you and your friends would like to reserve a group study room in the library at 2 PM this Wednesday. How would you make the room reservation using the library website?" In addition to the Penn State University Libraries (PSUL) website, the participants would also be asked to perform the same task on the websites of three peer institutions. They were then given a short interview to share their opinions based on their experiences.

The advantage of such a study is that the participants become more aware of possible alternative designs after seeing and experiencing other interfaces, which expands their vocabulary in describing their perspectives and preferences. Therefore, it is important to provide a variety of options from other welldeveloped websites with different functions and layouts to draw out users' perspectives of a good design. The usability testing results were measured in task success and task completion time. The rankings of test sites and the in-person interviews provide self-reported data from the participants. In addition to comparing with peer institutions, the testing format was also used to compare between the existing design and mockups within PSUL.

This comparative method was used to gauge users' perceptions of the top navigation menus, frequently used pages, and search result pages for the Discovery tool of the PSUL website. It was evident that a small design decision can trigger users' different reactions towards the interface. Though the scope of each study is discrete due to the time constraints of the UX Café, we have learned much about users' perspectives, which help guide future interface design decisions for the library's website. In the following sections, I will first describe the processes and results of three case studies and then discuss collectively how these comparative analyses help us gain insight on user experiences beyond usability.

\section{Case Study 1: Top Navigation Menu Context and Method}

In January 2015, PSUL started its site migration which was overseen by the Web Implementation Management Team (WIMT). Stakeholders debated whether to use drop-down menus or landing pages for the presentation of the top navigation. Due to time constraints, it was decided to move forward with landing pages and to conduct user studies afterwards. (In hindsight, such debates could be easily resolved by similar comparative analysis with peer institutions' navigation menus at the predesign stage.)

After migration was completed, a comparative analysis was conducted to gauge users' preference on the design of top navigation menus. The goal of the study was to investigate whether users prefer drop-down menus or landing pages. Also, should we include descriptions for the menu items? (See Figure 1 for an example from Northwestern University Libraries.) Three peer institutions-Northeastern University (NEU), Northwestern University (NWU), and North Carolina State University (NSCU)-were selected to compare based on their menu designs and functions (see Table 1). 
Figure 1. Top navigation menu of Northwestern University Libraries. The descriptions of the priority menu items append after the link.

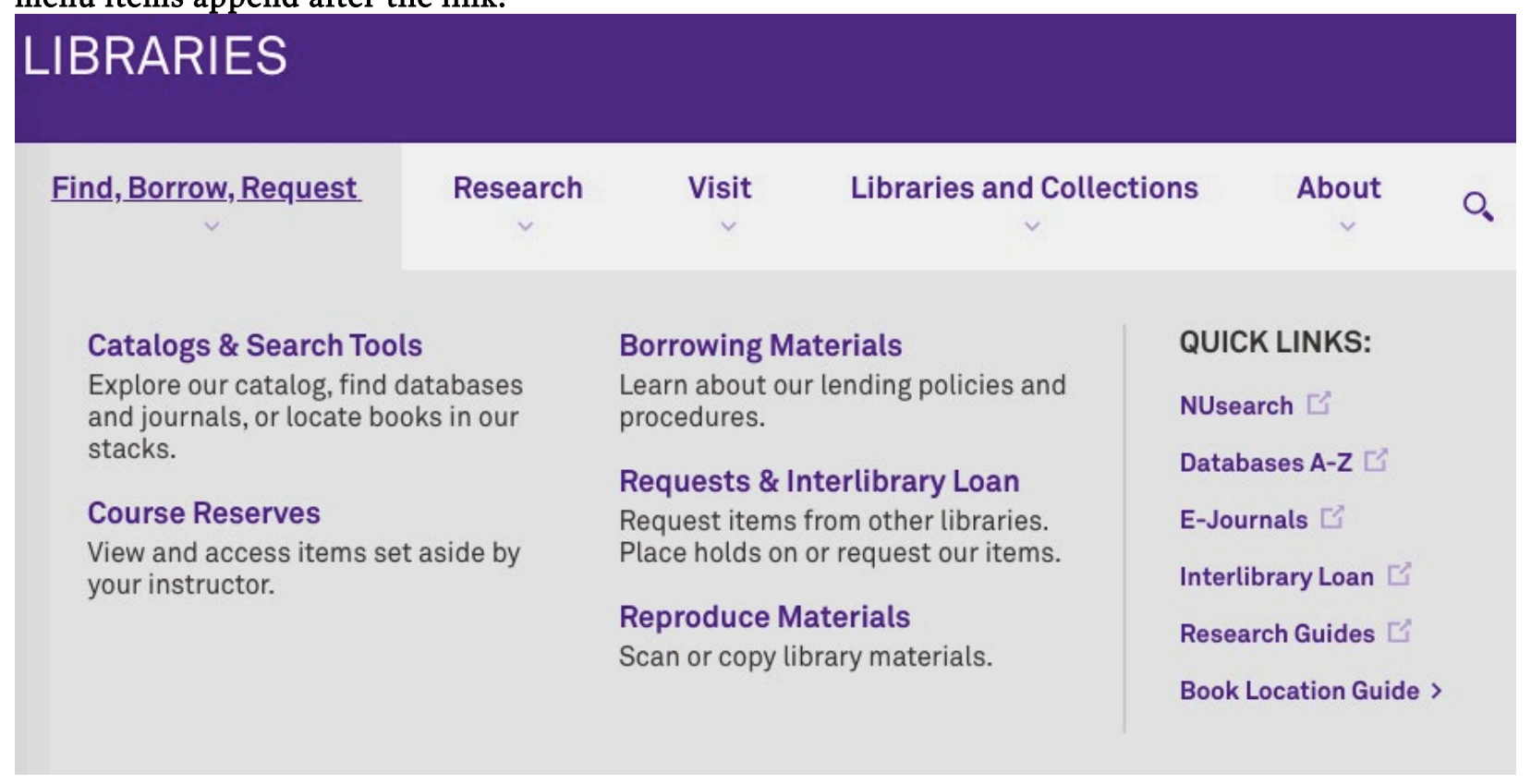

Table 1. Components of top navigation menu of selected libraries.

\begin{tabular}{|c|c|c|}
\hline & Function & Design \& Content \\
\hline NEU & Click to dropdown menu & Small, bolded links with descriptions \\
\hline NWU & Hover to dropdown menu & $\begin{array}{l}5-6 \text { prominent links with descriptions, plus a list of quick } \\
\text { links on the side }\end{array}$ \\
\hline NCSU & $\begin{array}{l}\text { Hover to dropdown menu, with a } \\
\text { 1-second delay }\end{array}$ & Regular links, without descriptions \\
\hline PSU & Click to landing page & Big, bolded links, with descriptions \\
\hline
\end{tabular}

Participants were asked to find the Interlibrary Loan (ILL) information of these three libraries' home pages using the top menu. They then were asked to complete the same task on Penn State Libraries' home page and to talk about their preferred design. They were told they could give up the task and move on to the next site if they thought they could not find the ILL page.

\section{Results}

Seventeen students participated in the study. Sixteen participants preferred hover to drop-down and one preferred the landing page. One student shared his insight that drop-down menus work better than a landing page when the Internet is slow. Most students (11 out of 17) did not read the description under the links. They admitted that they only scanned the anchor text and ignored the non-link text. Half of the participants (9 out of 17) were observed navigating back and forth between menus as they were uncertain which menu (e.g., Services, Research, or About) the interlibrary loan would fall into.

Not all students successfully found the ILL pages. Due to the task asking for "interlibrary loan" information, participants scanned for the term specifically. NWU has the term "interlibrary loan" in the menu as a link; NEU has it in the description; NCSU does not include the term in the menu. In the end, fifteen students 
found the interlibrary loan pages of NWU, eight for NEU, and one for NCSU. The majority (13 out of 17) preferred NWU's menu because they were able to scan the links easily for the term and complete the task successfully. Though many (11 out of 17) did not read the descriptions, they considered others would find the descriptions helpful and stated that they did not mind having the extra text in place.

\section{Case Study 2: Discovery Interface Context and Method}

A discovery tool, such as Serial Solution's Summon or EBSCO Discovery Services (EDS), offers users one search box and a unified interface for search results. Though most students prefer this Google-like one-stop search premise, many do not understand the search scope and expect to find libraries' local web pages in the result. To mitigate such confusion, NCSU Libraries first introduced the "bento-box layout" that presents multiple groupings to help students differentiate various libraries' collections and services. ${ }^{12}$

As more and more libraries adopted the bento-box approach, we wanted to know: are Penn State students satisfied with the results our discovery service, Summon, provides? Would they consider the bento-box layout helpful? What else can we learn from discovery services at other libraries? A comparative analysis was conducted to investigate users' perspectives and preferences for the front-end design of discovery services.

In order to provide a variety of looks and feels, I chose three peer institutions based on the interface of their discovery services to compare:

- Bento-box interface: Cornell University (https://www.library.cornell.edu)

- Primo interface: University of Oregon (http://library.uoregon.edu/)

- Customized interface for a cohesive look and feel with the library website: Brigham Young University (https://lib.byu.edu/)

Participants were asked to think of an upcoming assignment and come up with a query to search library resources for the assignment. They would conduct the search on PSUL's homepage (i.e., Summon), plus the three discovery services mentioned above.

\section{Results}

Five students participated in the study (one freshman, two juniors, and two graduate students). Overall, they all found useful articles or books for their assignments from the library websites; thus, there was no clear winner regarding relevancy. However, four out of five preferred the bento-box's clear grouping of content type (e.g., books, journal articles) over a long list of search results with facets on the side. Content type was an important factor in their evaluation of result pages, as two students were looking for articles and two for books. Students found it very helpful that the bento-box layout pointed them directly to the desired content type. The reason that one student did not favor bento-box design was because she saw only one book listed in the "Books" group of Cornell's bento-box, compared to others' listings of thousands. In addition to the bentobox design, students expressed preferences for book cover thumbnails and prominent essential item information (i.e., availability, call number, and item location).

\section{Case Study 3: Bento-Box Design Context and Method}

After my recommendation for implementing a bento-box interface, a discovery project team was formed to carry out the implementation. To further refine what design qualities students would find appealing about the interface, I followed up with a second comparative analysis focusing only on bento-box layouts. The discovery layer of three institutions-Cornell, Duke, and Princeton-were chosen to compare with the one of Penn State. Similar to the previous studies, participants were asked to perform tasks and evaluate the search result pages. Participants also ranked the bento-box interfaces in these three categories: labeling (the grouping and naming), layout (the design), and result (the relevancy and usefulness). In addition to the testing at UX Café, we created a paper-based survey (see Figure 2) and sent it to campus libraries with the goal of the study and instructions for how to administer the study. 
Figure 2. The paper survey for bento-box interface. The survey took the Olympics theme because it took place not long after the 2018 Winter Olympics.

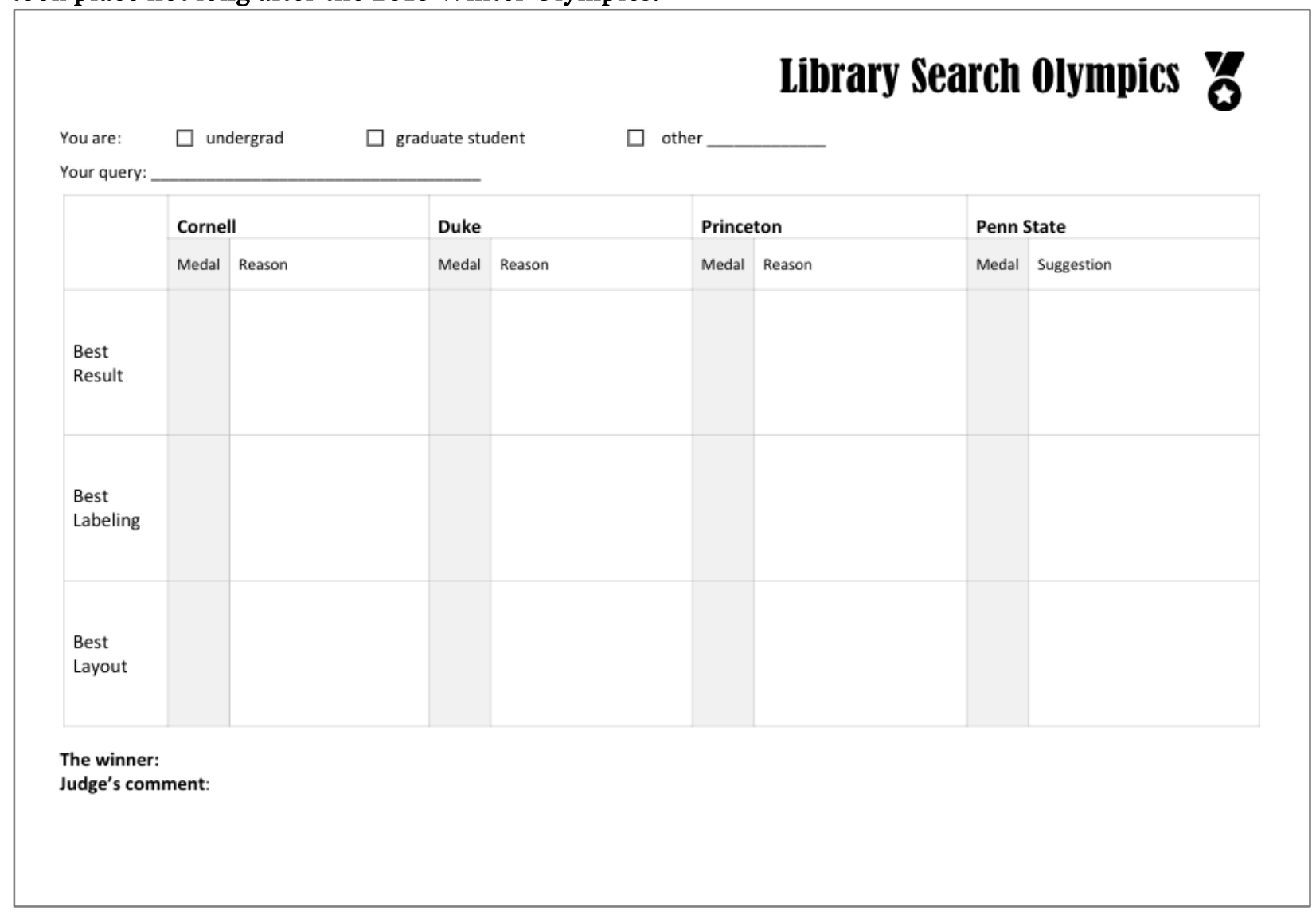

\section{Results}

A total of 59 participants completed the study, including six testing at the UX Café. Not all participants commented on the reasons behind each ranking. In addition, feedback like "easy to use" or "user-friendly" does not provide concrete reasons as to what makes it easy or friendly. Users' judgement on the interface and content is subjective; nevertheless, their preferred qualities emerged after comments were analyzed. Cornell's bento-box outperformed the rest in all three categories. The feedback shows that most participants favor Cornell for its clean and organized look for layout, refined and unambiguous grouping (for example, books and videos are in separate groups, not grouped together as one "Books and Media") for labeling, and the comprehensiveness and the left format filter for results.

\section{Discussion}

\section{Show the Possibilities}

In usability testing, the product is measured by participants' task performance. Participants' opinions outside of usability are often not investigated. Vaughn and Callicote gave participants a take-home evaluation with open questions about their opinions of the library website. The responses turned out to be generally positive but "vague and evasive." 13 The responses from my non-comparative usability testing paint a similar picture. "Straightforward" and "easy to use" are two common descriptors in the responses which are very abstract and not that helpful. There are many reasons why students are shy on giving specific suggestions. It could be that they are afraid that they might sound stupid because they are not familiar with library resources, or they feel they should not say anything negative because it might hurt librarians' feelings, or they have not even thought about how the website could be made better. 
The individual experience of existing products will influence and constrain his/her expectations about the possibilities. ${ }^{14}$ When doing usability testing on only one site, a user's focus is limited to the specific context and they may not think beyond that. In the case study of our top navigation menu, without looking at other libraries' navigation menus, participants are likely to concentrate only on the usability task and not think about other possibilities, such as dropdown menus. Also, considering they have likely never seen a bento-box layout, the chance of telling you that the bento-box is their preferred interface is almost zero.

\section{Learn the Positives}

By comparing the different designs and functions with other libraries, participants get a better sense on what a library website can offer. They become more articulate based on the examples in front of them. In helping improve our library website, they are open about what features they like and what we should adopt (for example, the book cover thumbnail). They are also candid about the less preferable features on other websites. The tone of the feedback changes from uncertainty to sharing personal preferences. The nature of the study shifts from merely preventing usability problems to creating positive experiences for users.

It is interesting to find out users do not mind having a feature they do not need on the interface as long as the feature does not hinder them in achieving their goals. For example, for the top navigation menu, many participants do not mind having description text next to the links even though they paid no attention to it. In the end, participants preferred Northwestern's top navigation menu because they were able to scan the links easily even with the descriptive text. They did not favor NCSU's menus, though the menus are clean and without extra text. The human brain is malleable. We have unconsciously learned to selectively take in information around us. The design of an interface is more than just minimizing the content to decrease the cognition load. It is also about how we facilitate the interaction between humans and computers. In this case, Northwestern uses strong design and labels to direct users' attention. As Hassenzahl, Law, and Hvannberg put it, "absence of the negative" does not necessarily equate with "positive." 15

Many positive comments for Cornell's bento-box design in terms of labels and results are generated by the granularity in grouping and the format filter. Not every library can invest the backend development work to support complex functions as such on the front end. However, the design attributes that users desire for a clean and organized interface-uncluttered, nicely spaced, and clearly aligned-are something we can and should work on. We can learn and adopt these positives-in this case, good design elements-from our peer institutions through comparative analysis.

\section{Take in Individual Context}

UX is complex. The pragmatic value and the affective value are often intertwined. A participant's reception of a product can be greatly influenced by a one-time execution of the product. For example, in case study 2 , the only participant who did not favor the bento-box layout for discovery layer did so because only one book was found with her query, in contrast to thousands of book results found on other platforms. A similar situation also happened in case study 3 , bento-box design. One participant strongly favored a less popular interface because her needed database was displayed prominently in the result compared to the results from other discovery layers. In both cases, their liking or disliking of an interface hinged on their context (i.e., the query they used) and, consequently, the product performance (i.e., the search results). As a result, it impacts the credibility of the service we provide and the loyalty from our patrons. They may have completely different opinions if they use different queries. It is very subjective. But this is the kind of nuance that may not be observed in a simple usability testing.

\section{Conclusion}

In User Experience-towards a unified view, Hassenzahl, Law, and Hvannberg presented three distinctions between UX study and usability study: holistic, subjective, and positive. ${ }^{16}$ As a holistic practice, UX looks for the interrelation between pragmatic aspects and hedonic aspects, taking the experience as a whole. UX is subjective because it takes into account that testing is an individual experience heavily depending on the context, and in many ways, user preference is as important as usability and other pragmatic measures. 
Moreover, UX is positive in looking beyond the focus of the usability problems and their removal and exploring the positive aspect of the interaction.

Nowadays, users are learning and adopting new technologies at an unprecedented speed. Their expectations for the web environment continue to evolve. Creating positive online user experiences should be a critical part of the library's ongoing endeavor. Comparative analysis can help us recognize the complex perspectives of our users and be our first steps in moving beyond usability and to the realm of UX.

-Copyright 2019 Zoe Chao

\section{Endnotes}

1. Jakob Nielsen, "The usability engineering life cycle," Computer 25, no.3 (1992): 12-22, http://doi.ieeecomputersociety.org/10.1109/2.121503.

2. Brian Shackel, "Usability-context, framework, definition, design and evaluation," Interacting with Computers 21, no. 5-6 (December 2009): 339-346, https://doi.org/10.1016/j.intcom.2009.04.007.

3. Martin Maguire and Nigel Bevan, "User requirements analysis," in Usability (Boston: Springer, 2002), 133148, https://doi.org/10.1007/978-0-387-35610-5_9; Jesmond J. Allen and James J. Chudley, "Gaining useful insight from competitor benchmarking," in Smashing UX design: Foundations for designing online user experiences (Hoboken: Wiley, 2012), 97-104.

4. Andrew D. Asher, Lynda M. Duke, and Suzanne Wilson, "Paths of discovery: Comparing the search effectiveness of EBSCO Discovery Service, Summon, Google Scholar, and conventional library resources," College \& Research Libraries 74, no. 5 (2013): 464-488, https://doi.org/10.5860/crl-374.

5. Rice Majors, "Comparative user experiences of next-generation catalogue interfaces," Library Trends 61, no. 1 (2012): 186-207, https://doi.org/10.1353/lib.2012.0029.

6. Craig M. MacDonald and Michael E. Atwood, "Changing perspectives on evaluation in HCI: past, present, and future," in CHI'13 extended abstracts on human factors in computing systems (ACM, 2013), 1969-1978, https://doi.org/10.1145/2468356.2468714.

7. Jakob Nielsen, "Guerrilla HCI: Using discount usability engineering to penetrate the intimidation barrier," https://www.nngroup.com/articles/guerrilla-hci/.

8. Thomas Tullis and Bill Albert, Measuring the user experience: collecting, analyzing, and presenting usability metrics (Morgan Kaufmann, 2013), 41-62.

9. Javier A. Bargas-Avila and Kasper Hornbæk, "Old wine in new bottles or novel challenges: a critical analysis of empirical studies of user experience," in Proceedings of the SIGCHI conference on human factors in computing systems (ACM, 2011), 2689-2698, https://doi.org/10.1145/1978942.1979336.

10. Marc Hassenzahl and Noam Tractinsky, "User experience-a research agenda," Behaviour \& information technology 25, no. 2 (2006): 91-97, https://doi.org/10.1080/01449290500330331.

11. Effie Law, Virpi Roto, Arnold P.O.S. Vermeeren, Joke Kort, and Marc Hassenzahl, "Towards a shared definition of user experience," in CHI'08 extended abstracts on Human factors in computing systems (ACM, 2008), 2395-2398, https://doi.org/10.1145/1358628.1358693.

12. Cory Lown, Tito Sierra, and Josh Boyer, "How users search the library from a single search box," College \& Research Libraries 74, no. 3 (2013): 227-241, https://doi.org/10.5860/crl-321.

13. Debbie Vaughn and Burton Callicott, "Broccoli librarianship and Google-bred patrons, or what's wrong with usability testing?" College \& Undergraduate Libraries 10, no. 2 (2004): 1-18, https://doi.org/10.1300/J106v10n02_01.

14. Wendy Olphert and Leela Damodaran, "Getting what you want, or wanting what you get? Beyond user centred design," in Design and Emotion (CRC Press, 2004), 138-143.

15. Marc Hassenzahl, Effie Law, and Ebba Thora Hvannberg, "User Experience-Towards a unified view," in Proceedings of Int'l COST294-MAUSE Workshop, October 14, Oslo, Norway (UX WS NordICHI, 2006), 1-3.

16. Hassenzahl, Law, and Hvannberg, "User Experience." 